\title{
Manifestaciones neurológicas del lupus eritematoso sistémico: Revisión de literatura
}

\author{
Neurological manifestations of systemic lupus erythematosus: \\ A comprehensive literature review
}

Eduardo Vásconez-González, María Belén-López, Dayana Cuchiparte, Katherine Peláez, Claudio Galarza-Maldonado,

Leonardo Cano-Cevallos, ${ }^{1}$ Andrés López-Cortés, ${ }^{3,4}$ Esteban Ortiz- Prado ${ }^{1}$

\begin{abstract}
Resumen
Dentro de las enfermedades autoinmunes destaca el lupus eritematoso sistémico (LES). Esta enfermedad está caracterizada por la afectación de varios órganos y sistemas, entre ellos el sistema nervioso central, en el cual produce diversas complicaciones, algunas poco comunes o documentadas en la literatura lo que dificulta su diagnóstico, y que conlleva a demoras en su tratamiento, pues muchas de estas ocurren después del diagnostico inicial de LES, y es la razón por la que se recomienda la valoración neurológica durante el seguimiento de pacientes con esta enfermedad.
\end{abstract}

Palabras clave: SNC, LES, Complicaciones neurológicas

\section{Abstract}

Among autoimmune diseases, systemic lupus erythematosus (SLE) stands out. This disease is characterized by the involvement of several organs and systems, including the central nervous system, in which it produces various complications, some rare or documented in the literature, which makes its diagnosis difficult, resulting in delays for its treatment. Many of these complications occur after the initial diagnosis of SLE; for that reason, a neurological evaluation is recommended in the followup of patients with SLE.

Keywords: SLE, CNS, Neurologic complications

Rev. Ecuat. Neurol. Vol. 30, N² 2, 2021

\section{Introducción}

Las enfermedades autoinmunes ocurren cuando el sistema inmunológico ataca a las moléculas propias como resultado de una disrupción en la tolerancia inmunológica hacia las células inmunes autorreactivas. Este tipo de trastornos tienen una prevalencia del 3-5\% a nivel mundial. En el caso de lupus eritematosos sistémico (LES), la prevalencia oscila entre $10-150$ casos por cada 100.000 habitantes.,3 Esta enfermedad tiene varios fenotipos, con presentaciones clínicas variables que van desde manifestaciones mucocutáneas leves hasta afectación multiorgánica grave, que dentro de ellos incluye al sistema nervioso central y periférico.

La prevalencia de manifestaciones neuropsiquiátricas es del $30-40 \%$ en pacientes diagnosticados con LES, donde se estima que en un 14-80\% ocurre en adultos, y
$22-95 \%$ en niños. ${ }^{3,5}$ Los factores de riesgo identificados que predisponen al desarrollo de estas manifestaciones incluyen la actividad sistémica del LES y extensión del daño de órganos (sin incluir SNC) bajo tratamiento con altas dosis de esteroides, presencia previa de eventos neuropsiquiátricos, y por último, presencia de anticuerpos antifosfolípidicos persistentemente positivos. ${ }^{3}$ Otros factores secundarios importantes a considerar sugieren que el $50-78 \%$ de eventos neurológicos pueden estar asociados a infecciones por terapia immunosupresora, complicaciones metabólicas por fallo de órganos como uremia, hipertensión, y toxicidad por tratamiento (corticoesteroides).

Existe un amplio espectro de condiciones neurológicas que pueden ocurrir en un paciente con LES, y que han sido clasificadas principalmente por su aparición, tanto
'One Health Research Group, Universidad de las Americas, Facultad de Medicina, Quito, Ecuador.

${ }^{2}$ Unidad de Enfermedades Reumáticas y Autoinmunes-UNERA, Corporación Médica Monte Sinaí, Cuenca, Ecuador.

${ }^{3}$ Centro de Investigación Genética y Genómica, Facultad de Ciencias de la Salud Eugenio Espejo, Universidad UTE, Quito, Ecuador.

${ }^{4}$ Latin American Network for the Implementation and Validation of Clinical Pharmacogenomics Guidelines (RELIVAF-CYTED), Madrid, Spain.
Correspondencia:

Esteban Ortiz-Prado, M.D., Ph.D.

One Health Research Group, Universidad de las Americas, Quito, Ecuador Calle de los Colimes y Avenida De los Granados, Quito 170137, Ecuador.

E-mail: e.ortizprado@gmail.com

Teléfono: +593995760693 
como una manifestación neurológica primaria de la enfermedad, o como una complicación secundaria de la misma, sea por el nivel de compromiso sistémico, la cronicidad de la enfermedad o también por algun efecto del tratamiento. Cabe destacar que en esta revisión, su enfoque es específico a las manifestaciones y complicaciones neurológicas, y no psiquiatricas causadas por el lupus eritematoso sistémico (Tabla 1).

Tabla 1. Clasificación de manifestaciones neurológicas en el sistema nervioso causadas por el lupus eritamtoso sistémico? (Adaptado de la nomenclatura de ACR para definición de casos de síndromes neuropsiquiátricos lúpicos)

\begin{tabular}{|ll|}
\hline Sistema nervioso central & Sistema nervioso periférico \\
\hline Enfermedad cerebrovascular & Síndrome Guillain-Barre \\
\hline Síndromes desmielinizantes & Neuropatía autonómica \\
\hline Meningitis aséptica & Mononeuropatias \\
\hline Mielopatía & Miastenia gravis \\
\hline Cefalea & Neuropatía craneal \\
\hline Convulsiones & Plexopatías \\
\hline Trastornos de movimiento & Polineuropatías \\
\hline Confusión & \\
\hline Disfunción cognitiva & \\
\hline Ansiedad/depresión & \\
\hline Trastornos del ánimo \\
\hline Psicosis \\
\hline
\end{tabular}

\section{Síndromes neurológicos asociados a lupus} eritematoso sistémico

Eventos cerebrovasculares

Accidente cerebrovascular isquémico

Las personas diagnosticadas con LES tienen un mayor riesgo de sufrir accidentes cerebrovasculares isquémicos; se estima que alrededor del 3-20\% de las personas con LES se ven afectadas dentro de los primeros 5 años de diagnóstico, así, hay datos que atribuyen a la enfermedad cerebrovascular (ECV) como responsable de un $10-15 \%$ de las muertes en pacientes con esta enfermedad. ${ }^{9}$

Existen numerosos mecanismos involucrados en la fisiopatología de la ECV relacionados con LES, que incluyen estados de hipercoagulabilidad, embolia cardiogénica, ateroesclerosis prematura o acelerada, vasculitis, formación de autoanticuerpos contra antígenos neuronales, ribosomales y fosfolipídicos, y producción intratecal de mediadores inflamatorios ${ }^{3,10}$ En algunos casos esta entidad puede ser la manifestación de presentación del LES, y se ha visto más comúnmente en pacientes jóvenes, en especial mujeres, afectando con mayor predilección al territorio vertebrobasilar. ${ }^{10,11}$

Los signos o síntomas sugestivos de un evento cerebrovascular, incluyen déficit motor con incapacidad para combinar movimientos, afasia, confusión, trastorno del equilibrio y mareo. Para su diagnóstico, la tomografía computarizada tiene una buena sensibilidad para las lesiones hemorrágicas a diferencia del ictus isquémico, no obstante la resonancia magnética tiene mayor sensibilidad en la detección de lesiones cerebrales relacionadas con la afectación cerebrovascular en general. ${ }^{12}$

El tratamiento es similar al de las personas sin LES. Este incluye trombólisis o cirugía según sea la indicación, más el control de factores de riesgo en la prevención secundaria. Adicionalmente, en los pacientes con lupus se debe de evaluar necesidad de anticoagulación o antiagregación ante la presencia de anticuerpos anticardiolipinas o del uso de esteroides e inmunosupresores ante la actividad severa del LES?

\section{Trombosis del seno venoso cerebral}

La trombosis del seno venoso cerebral (TSVC) ha sido raramente reportada en la literatura. Se ha informado que se presenta en alrededor 0.4-6.6\% de los pacientes con LES. Una de las teorías sobre la causa de la TSVC es la lesión de las células endoteliales causada por vasculitis inducida por complejos inmunes o debido a anticuerpos antifosfolípidos. ${ }^{13,14}$

Los síntomas más comunes que han sido descritos son cefalea, náuseas, vómito, alteración de la conciencia, edema palpebral, visión borrosa, diplopía y convulsiones. ${ }^{13}$ La venografía por resonancia magnética es el estudio más sensible para su diagnóstico. En cuanto a la punción lumbar para el análisis de líquido cefalorraquídeo, esta suele ser inespecífica pero es de utilidad para diferentes infecciones. ${ }^{13}$ La TSVC se trata con anticoagulación mediante heparina no fraccionada o heparina de bajo peso molecular seguida de warfarina con un objetivo de INR de 2-3.

\section{Síndrome de encefalopatía posterior reversible}

El síndrome de encefalopatía posterior reversible (PRES) se ha podido documentar en 0.4-1.8 \% de los pacientes con LES. Es una entidad clínicoradiológica caracterizada por cefalea, obnubilación y ceguera cortical asociada a edema cerebral en neuroimagen. ${ }^{15,16} \mathrm{Su}$ incidencia es desconocida, habiéndose reportado en un rango amplio de edad de 14 a 78 años, con una edad media de 44 años $\mathrm{y}$ una proporción hombre/mujer de $0.8 / 1 .^{16}$

La fisiopatología del PRES no es clara, pero se maneja la teoría de que el incremento súbito de la presión arterial supera la autorregulación del flujo sanguíneo cerebral, causando vasodilatación e hipoperfusión, con rotura subsiguiente de la barrera hematoencefálica y edema vasogénico. ${ }^{16}$

El diagnóstico diferencial del PRES incluye infartos en el territorio de la arteria cerebral posterior, trombosis venosa, trastornos desmielinizantes, vasculitis y encefalitis. ${ }^{15} \mathrm{Su}$ diagnóstico se realiza a través de estudios de imagen como la tomografía computarizada o la resonancia magnética. ${ }^{15,16}$

\section{Mielitis transversa}

La mielitis transversa aguda (MTA) es una inflamación aguda de la sustancia gris y blanca en uno o más seg- 
mentos adyacentes de la médula espinal. Esta es una rara complicación en los pacientes con LES que afecta en 1-2\% de estos pacientes. ${ }^{17,18} \mathrm{El}$ mecanismo fisiopatológico es incierto, aunque se ha sugerido la presencia de vasculitis y trombosis arterial, que provocan necrosis isquémica del cordón espinal. ${ }^{17}$ También se cree que el daño neurológico puede ser causado por una combinación de células inflamatorias, autoanticuerpos, quimiocinas, y estrés oxidativo.

La presentación clínica incluye alteraciones motoras sensoriales y también de esfínteres. Se puede acompañar de síntomas sistémicos como fiebre y otros signos de actividad de la enfermedad, con o sin compromiso de otros órganos. ${ }^{19}$

La resonancia magnética se considera el método de diagnóstico de elección para confirmar la MTA; el tratamiento de base es administrar altas dosis de corticoesteroides, combinados o no con inmunosupresores y plasmaféresis.17,18

\section{Leucoencefalopatia multifocal progresiva}

Reportes de leucoencefalopatía multifocal progresiva han sido publicados con cifras de 1.0-2.4 por cada100,000 casos de LES. Su causa está relacionada a la reactivación del virus JC (JCV), un poliomavirus humano ${ }^{8,20}$ Las principales manifestaciones incluyen alteraciones visuales, debilidad motora, estado mental alterado y déficit sensorial; ${ }^{20}$ para su diagnóstico la resonancia magnética es el método de imagen más sensible para la investigación; también se puede identificar el virus JC o sus anticuerpos en el suero, aunque el diagnóstico definitivo requiere de una biopsia cerebral., ${ }^{8,20} \mathrm{El}$ tratamiento está basado en la inmunomodulación y la terapia antiviral., ${ }^{8,20}$ (Tabla 2).

\section{Neuropatias}

\section{Neuropatía periférica}

Se ha reportado polineuropatía en $3.4-7.5 \%$ de los pacientes con LES. La polineuropatía periférica más común es la de tipo sensorial en un $36.7 \%$, seguida de la

Tabla 2. Manifestaciones y complicaciones neurológicas de lupus eritematoso sistémico?

\begin{tabular}{|c|c|}
\hline $\begin{array}{l}\text { Manifestaciones } \\
\text { neurológicas primarias }\end{array}$ & $\begin{array}{l}\text { Sistema nervioso periférico } \\
\text { Complicaciones secundarias } \\
\text { (enfermedad avanzada, tratamiento) }\end{array}$ \\
\hline $\begin{array}{l}\text { Enfermedad cerebrovascular } \\
\text { - Vasculitis } \\
\text { - Vasculopatía (aterosclerosis) } \\
\text { - Embolia } \\
\text { - Anticuerpos antifosfolípidos }\end{array}$ & $\begin{array}{l}\text { Enfermedad cerebrovascular } \\
\text { Convulsiones } \\
\text { Meningitis }\end{array}$ \\
\hline Mielitis transversa & \\
\hline Neuritis óptica & \\
\hline Meningitis & \\
\hline Neuropatías & \\
\hline Coma & \\
\hline Migraña & \\
\hline Convulsiones & \\
\hline Síndrome cerebral orgánico & \\
\hline
\end{tabular}

variante sensitivo-motora en $18.8 \%{ }^{21}$ En el $17.1 \%$ pacientes con neuropatía periférica, estos manifiestan una neuropatía de fibras pequeñas, la cual tiene distintas entidades clínico-patológicas. De todas ellas, la primera que se ha caracterizado es la neuropatía de fibras pequeñas dependiente de la longitud, la cual presenta una distribución de dolor neuropático en media-guante y tiene hallazgos anormales de biopsia de piel restringidos a la parte distal de la pierna. La segunda se denomina neuropatía de fibras pequeñas no dependiente de la longitud; en esta el dolor neuropático es irregular, asimétrico y proximal, que puede afectar la cara, el torso y las extremidades proximales y se observa hallazgos anormales de la biopsia de piel en la parte proximal del muslo. ${ }^{22} \mathrm{El}$ tratamiento de las neuropatías está basa en corticoesteroides e inmunosupresión.,23

\section{Neuropatías craneales}

La neuropatía craneal ha sido descrita en 1.7-2.4\% de pacientes con LES; la parálisis aislada del nervio motor ocular común es un tipo de neuropatía craneal que se observa con el LES y por lo general la neuropatía periférica se desarrolla en el $0.1 \%$ de los pacientes durante el período anterior al diagnóstico de LES.24 Pueden verse afectados un solo nervios o varios nervios a la vez, los más comunes en son III, V, VI y VII?

\section{Neuritis óptica}

La neuritis óptica se ha reportado en el 1\% de los pacientes diagnosticados con LES $;^{8}$ esta puede estar asociada con anticuerpos anti-acuaporina-4 que han demostrado causar citotoxicidad in vitro en astrocitos. También está implicado un proceso de vasculitis oclusiva que afecta a las pequeñas arteriolas del nervio óptico, lo que conduce a una necrosis axonal, ${ }^{25,26}$ está caracterizada por la presencia de escotoma central, pérdida progresiva de la visión, dolor orbitario y ceguera. ${ }^{8} 25$ El diagnóstico se realiza clínicamente, y los estudios de imagen como la resonancia magnética muestran realce del nervio óptico. El manejo se da mediante administración de corticoides a altas dosis . $^{825}$

\section{Miopatía necrotizante autoinmune}

Se estima que alrededor del $21 \%$ de pacientes con miopatía necrotizante autoinmune tiene LES $;^{8}$ esta se presenta con debilidad simétrica predominantemente proximal de miembros superiores e inferiores, de inicio tipo agudo o subagudo, junto con niveles altos de creatina quinasa y hallazgos miopáticos anormales en la electromiografía. ${ }^{27}$

\section{Meningitis aséptica}

La meningitis es una manifestación rara, observada en $1.4-2.0 \%$ de los pacientes con LES. Esta puede ser séptica o aséptica, siendo casi el $50 \%$ del tipo aséptica: ${ }^{28}$ las manifestaciones clínicas incluyen fiebre, cefalea, signos meníngeos, pleocitosis en líquido cefalorraquídeo y 
cultivos negativos; varios informes han asociado el uso de antinflamatorios no esteroideos, especialmente ibuprofeno, con meningitis aséptica, ${ }^{3,8}$ Para su diagnóstico es indispensable el análisis de líquido cefalorraquídeo. Una vez confirmado el diagnóstico, el uso de corticoesteroides está indicado como tratamiento, además de medidas de soporte. ${ }^{28}$

\section{Enfermedad desmielinizante}

Esta entidad se ha reportado en el $0.3-2.7 \%$ de los pacientes con LES, la desmielinización puede tener una presentación monofásica o multifásica; esta cursa con aparición de lesiones inflamatorias focales en la sustancia blanca cerebral, provocando el funcionamiento anormal de las fibras nerviosas en las vías neuronales., ${ }^{3,8}$ Las características neuropatológicas encontradas son infartos multifocales y angiopatía no inflamatoria de pequeños vasos con proliferación de la íntima, fibrosis, hiperplasia mucoide y hialinización, tortuosidad de los vasos y gliosis perivascular..$^{29} \mathrm{El}$ tratamiento incluye metilprednisolona y ciclofosfamida intravenosa. En casos de recaídas se emplea terapia inmunosupresora de mantenimiento. Cuando el desarrollo de la enfermedad es más severo se puede emplear plasmaféresis. En el diagnóstico se emplean estudios de imagen como la resonancia magnética?

\section{Abordaje diagnóstico}

El LES puede comprometer el sistema nervioso causando distintas enfermedades. Sería de gran utilidad poder contar con pruebas diagnósticas que nos permitan identificar de manera específica cada una de las entidades antes mencionadas para establecer rápida y correctamente su diagnóstico, sin embargo no existen. Es por esto que el estudio de manera general en estos pacientes se centra en establecer el diagnóstico de LES, distinguirlo entre causa funcional u orgánica, y excluir aquellos síntomas que no sean atribuibles al lupus (Figura 1).

Existe un conjunto estudios que son apropiados para poder llegar al diagnóstico de estas patologías que son: estudios serológicos, análisis de líquido cefaloraquídeo (LCR), electroencefalografía, y estudios de imágenes (Tabla 3)

En cuanto al tratamiento de todas estas entidades clínicas, la mayoría son tratadas con el uso de corticosesteroides (prednisona, metilprednisolona) con el fin de contrarestar el proceso inflamatorio que esta causando la alteración a nivel del órgano afectado, sea nervios, músculos y vasos sanguíneos. La terapia anticoagulante toma un rol importante también, ya que disminuye el estado de hipercoagulabilidad al cual predispone el LES. Adicionalmente se pueden adicionar terapias específicas dependiendo de la enfermedad tales como hidroxicloroquina o plasmaféresis.

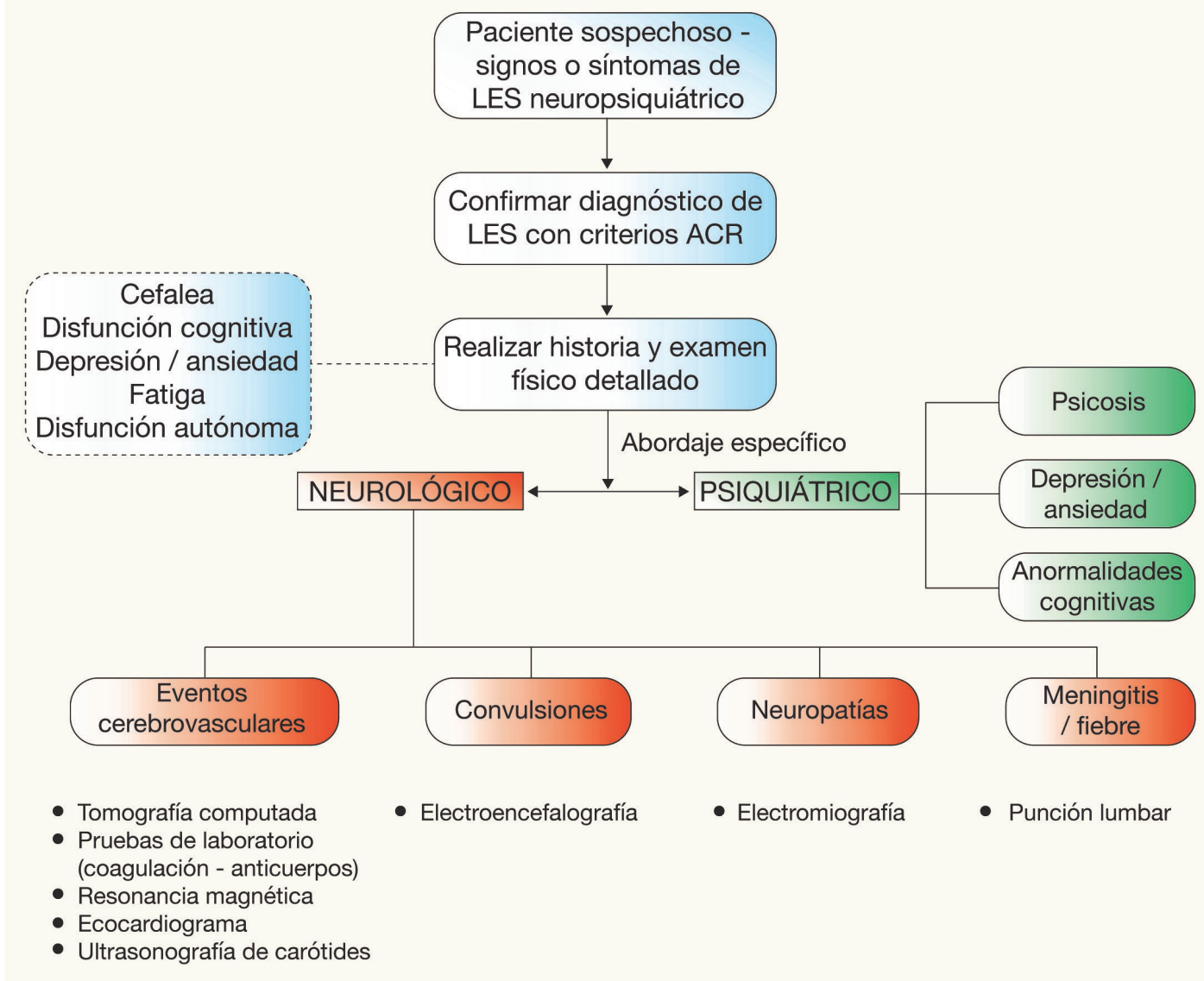

Flgura 1. Diagrama para la toma de decisiones en pacientes con LES y sospecha de afectación neurológica. (Elaboración propia) 
Tabal 3. Estudios diagnósticos útiles en pacientes con manifestaciones neurológicas por LES.

\begin{tabular}{|c|c|}
\hline Estudios serológicos & $\begin{array}{l}\text { Anticuerpos antinucleares (ANA): altamente sensible para el diagnóstico de LES. } \\
\text { Anticuerpos Anti-ADN - Anti-Sm: alta específicos, útiles para corroborar el diagnóstico de LES. } \\
\text { Niveles de complemento (C3-C4): útiles en el diagnóstico, compatible con proceso inmunológico y formación de inmunocomplejos. } \\
\text { Anticuerpos antifosfolípidos: si están presentes pueden asociarse a eventos cerebrovasculares, trombosis, mielitis transversa. }{ }^{30}\end{array}$ \\
\hline $\begin{array}{l}\text { Análisis en líquido } \\
\text { cefalorraquídeo }\end{array}$ & $\begin{array}{l}\text { Comúnmente el análisis de LCR es normal en pacientes con lupus y compromiso del CNS salvo en los siguientes casos: } \\
\text { - Meningitis aséptica } \\
\text { - Vasculitis } \\
\text { - Mielitis transversa } \\
\text { En algunos reportes se ha podido identificar ciertas anormalidades inmunológicas tales como niveles elevados de anticuerpos anti-ADN, } \\
\text { IgG, bandas oligoclonales, complejos inmunes, interleucina- } 6.31\end{array}$ \\
\hline Electroencefalografía & $\begin{array}{l}\text { Aproximadamente un } 80 \% \text { de pacientes con lupus en SNC activo tendrán resultados anormales por mapeo electroencefalográfico. } \\
\text { Típicamente mostrando encefalopatía, con actividad de ondas lentas difusas, frente a cambios focales que pueden ser vistos en } \\
\text { epilepsias u otros problemas neurológicos. } .^{32}\end{array}$ \\
\hline Estudios de imágenes & $\begin{array}{l}\text { Los métodos más útiles son la tomografía computarizada y resonancia magnética. } \\
\text { Tomografía computarizada: hábil en la detección de anomalias estructurales y focales como infartos, hemorragias, tumores, } \\
\text { calcificaciones, abscesos, meningitis. } \\
\text { Resonancia magnética: tiene una mayor sensibilidad para revelar anomalías focales, como también lesiones periventriculares o en } \\
\text { sustancia blanca. Se la recomienda particularmente en aquellos pacientes con trastornos neurológicos focales, convulsiones, disfunción } \\
\text { cognitiva crónica y síndrome antifosfolípidos con trastornos neurológicos. }{ }^{33}\end{array}$ \\
\hline
\end{tabular}

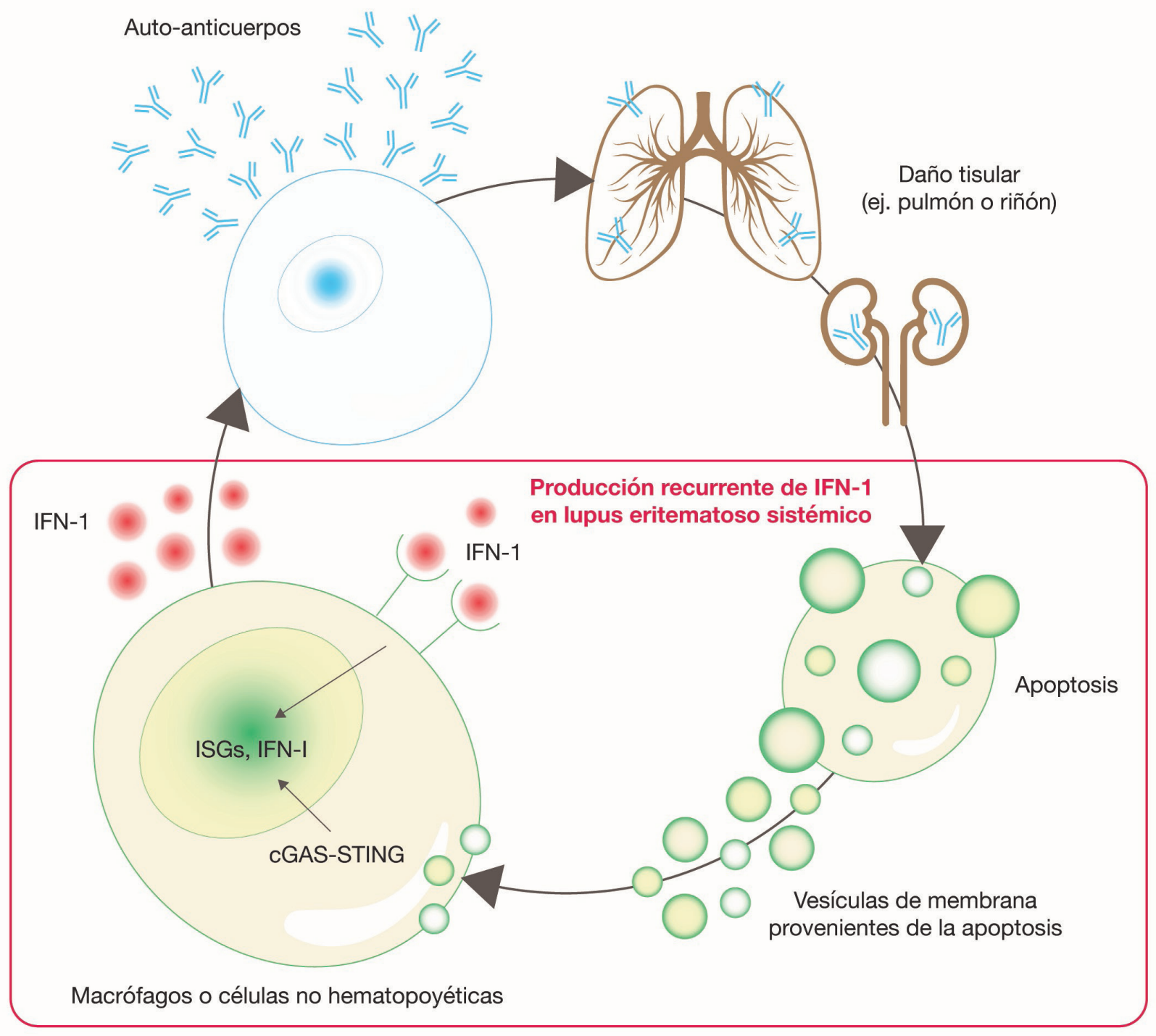

Figura 2. Fisiopatología propuesta de las implicaciones neurológicas del LES. (Elaboración propia) 
En casos refractarios o severos el uso de ciclofosfamida también se recomienda en ciertas patologías, debido a su buena respuesta. ${ }^{34}$

La realización de este trabajo tiene como fin sintetizar información acerca de las manifestaciones neurológicas causadas por el lupus eritematoso sistémico. Es importante señalar que muchas, en ocasiones, son la manera en que los pacientes con LES se manifiestan inicialmente con la enfermedad, y en otras ocasiones, ocurren tras el diagnóstico inicial de la misma. Dentro de la fisiopatología, muchas de estas manifestaciones o complicaciones no tienen una explicación científica clara, pero la mayoría se enmarca en las teorías de vasculitis, trombosis arterial, presencia de anticuerpos antifosfolípido, estados de hipercoagulabilidad, embolia cardiogénica y aterosclerosis prematura (Figura 2).

Las complicaciones pueden ser trastornos comunes como accidentes cerebrovasculares, y otros tan poco conocidos y documentados como miopatía necrotizante autoinmune, neuritis de tipo craneales o la encefalopatía posterior reversible, por lo cual es aconsejable que en pacientes que debuten con estas enfermedades de afectación nerviosa, se proceda a descartar LES como parte del diagnóstico diferencial, y realizar las pruebas pertinentes para un diagnóstico oportuno y atención médica necesaria; además en aquellos pacientes previamente diagnosticados con LES y sobre todo en aquellos que tengan antecedentes de eventos neuropsiquiátricos y anticuerpos antifosfolípidos persistentemente positivos, realizar seguimientos rigurosos y evaluar constantemente el sistema nervioso en busca de signos o síntomas de complicaciones para realizar un diagnóstico oportuno.

\section{Referencias}

1. Smith DA, Germolec DR. Introduction to immunology and autoimmunity. Environ Health Perspect. 1999 Oct;107(Suppl 5):661-5. https://dx.doi. org/10.1289\%2Fehp.99107s5661.

2. Wang L, Wang F-S, Gershwin ME. Human autoimmune diseases: a comprehensive update. J Intern Med. 2015;278(4):369-95. https://doi.org/10.1111/joim.12395

3. Díaz-Cortés D, Correa-González N, Díaz MC, Gutiérrez JM, Fernández-Ávila DG. Compromiso del sistema nervioso central en el lupus eritematoso sistémico. Rev Colomb Reumatol. 2015 Mar 1;22(1):16-30. http://dx.doi.org/10.1016/j.rcreu.2015.02.001

4. Justiz Vaillant AA, Goyal A, Bansal P, Varacallo M. Systemic Lupus Erythematosus. In: StatPearls [Internet]. Treasure Island (FL): StatPearls Publishing; 2020 [cited 2021 Jan 24]. Available from: http:// www.ncbi.nlm.nih.gov/books/NBK535405/

5. Muscal E, Brey RL. Neurological Manifestations of Systemic Lupus Erythematosus in Children and Adults. Neurol Clin. 2010 Feb;28(1):61-73. https://doi. org/10.1016/j.ncl.2009.09.004
6. Hanly JG, Su L, Farewell V, McCURDY G, Fougere L, Thompson K. Prospective Study of Neuropsychiatric Events in Systemic Lupus Erythematosus. J Rheumatol. 2009 Jul;36(7):1449-59. https://doi. org $10.3899 /$ jrheum.081133

7. ACR AD HOC COMMITTEE ON NEUROPSYCHIATRIC LUPUS NOMENCLATURE. The American College of Rheumatology nomenclature and case definitions for neuropsychiatric lupus syndromes. Arthritis Rheum. 1999 Apr;42(4):599-608. https://doi. org/10.1002/1529-0131(199904)42:4\%3C599::AIDANR2\%3E3.0.CO;2-F

8. Shaban A, Leira EC. Neurological Complications in Patients with Systemic Lupus Erythematosus. Curr Neurol Neurosci Rep. 2019 Nov 26;19(12):97. https:// doi.org/10.1007/s11910-019-1012-1

9. Holmqvist M, Simard JF, Asplund K, Arkema EV. Stroke in systemic lupus erythematosus: a meta-analysis of population-based cohort studies. RMD Open. 2015 Dec 1;1(1):e000168. https://doi. org/10.1136/rmdopen-2015-000168. PMID: 26719816

10. Ioannidis S, Mavridis M, Mitsias PD. Ischemic stroke as initial manifestation of systemic lupus erythematosus: A case report and review of the literature. eNeurologicalSci. 2018 Nov 9;13:26-30. https://doi.org/10.1016/j.ensci.2018.11.001

11. Nikolopoulos D, Fanouriakis A, Boumpas DT. Cerebrovascular Events in Systemic Lupus Erythematosus: Diagnosis and Management. Mediterr J Rheumatol. 2019 Mar 28;30(1):7-15. https://doi. org/10.31138/mjr.30.1.7

12. de Amorim LCD, Maia FM, Rodrigues CEM. Stroke in systemic lupus erythematosus and antiphospholipid syndrome: risk factors, clinical manifestations, neuroimaging, and treatment. Lupus. 2017 Apr 1;26(5):529-36. https://doi. org/10.1177/0961203316688784

13. Wang L, Chen H, Zhang Y, Liu W, Zheng W, Zhang $\mathrm{X}$, et al. Clinical Characteristics of Cerebral Venous Sinus Thrombosis in Patients with Systemic Lupus Erythematosus: A Single-Centre Experience in China [Internet]. Vol. 2015, Journal of Immunology Research. Hindawi; 2015 [cited 2021 Jan 21]. p. e540738. https://doi.org/10.1155/2015/540738

14. Lee MK, Kim JH, Kang HR, Rho HJ, Nam EJ, Kim $\mathrm{SW}$, et al. Systemic lupus erythematosus complicated with cerebral venous sinus thrombosis: a report of two cases. J Korean Med Sci. 2001 Jun;16(3):351-4. https://dx.doi.org/10.3346\%2Fjkms.2001.16.3.351

15. Blanch García P, Moral Torres E, Marín Muñoz J, Corominas Macías H, Mallafre Anduig J. Síndrome de encefalopatía posterior reversible causada por emergencia hipertensiva en paciente con lupus. Hipertens Riesgo Vasc. 2010 Nov 1;27(6):259-63. https://doi.org/10.1016/j.hipert.2009.10.002 
16. Fuseau Herrera M, Villagómez Estrada M, Garrido Salazar D, Noboa Torres D, Escudero Abad L, Narváez Castillo $\mathrm{B}$. Diagnóstico y manejo de síndrome de encefalopatía posterior reversible asociado a lupus eritematoso sistémico. Reporte de caso clínico. Rev Colomb Reumatol. 2019 Jan;26(1):74-9. https://doi. org/10.1016/j.rcreue.2019.05.003

17. Kovacs B, Lafferty TL, Brent LH, DeHoratius RJ. Transverse myelopathy in systemic lupus erythematosus: an analysis of 14 cases and review of the literature. Ann Rheum Dis. 2000 Feb 1;59(2):120-4. https://doi.org/10.1136/ard.59.2.120

18. Velloso Feijoo ML, García Hernández F, Ocaña Medina C, González León R, Garrido Rasco R, Sánchez Román J. Transverse Myelitis in Systemic Lupus Erythematosus. Reumatol Clínica. 2007 Mar 1;3(2):73-7. https:// doi.org/10.1016/S2173-5743(07)70215-0

19. Hryb JP, Chiganer E, Contentti EC, Di Pace JL, Lessa C, Perassolo MB. Myelitis in systemic lupus erythematosus: clinical features, immunological profile and magnetic resonance imaging of five cases. Spinal Cord Ser Cases. 2016 Jul 7;2:16005. https://doi. org $/ 10.1038 /$ scsandc. 2016.5

20. Berntsson SG, Katsarogiannis E, Lourenço F, Moraes-Fontes MF. Progressive Multifocal Leukoencephalopathy and Systemic Lupus Erythematosus: Focus on Etiology. Case Rep Neurol. 2016 Mar 16;8(1):59-65. https://doi.org/10.1159/000444874

21. Florica B, Aghdassi E, Su J, Gladman DD, Urowitz MB, Fortin PR. Peripheral Neuropathy in Patients with Systemic Lupus Erythematosus. Semin Arthritis Rheum. 2011 Oct 1;41(2):203-11. https://doi. org/10.1016/j.semarthrit.2011.04.001

22. Oomatia A, Fang H, Petri M, Birnbaum J. Peripheral Neuropathies in Systemic Lupus Erythematosus: Clinical Features, Disease Associations, and Immunologic Characteristics Evaluated Over a TwentyFive-Year Study Period. Arthritis Rheumatol. 2014;66(4):1000-9. https://doi.org/10.1002/art.38302

23. Xianbin W, Mingyu W, Dong X, Huiying L, Yan X, Fengchun Z, et al. Peripheral Neuropathies Due to Systemic Lupus Erythematosus in China. Medicine (Baltimore). 2015 Mar;94(11):e625. https://doi. org/10.1097/MD.0000000000000625

24. Nakamagoe K, Yanagiha H, Miyake Z, Kondo Y, Hiyama T, Ishii A, et al. Monocular Oculomotor Nerve Disorder Manifesting as Cranial Neuropathy in Systemic Lupus Erythematosus. Intern Med. 2018 Dec 1;57(23):3445-9. https://doi.org/10.2169/internalmedicine.1106-18

25. de Andrade FA, Guimarães Moreira Balbi G, Bortoloti de Azevedo LG, Provenzano Sá G, Vieira de
Moraes Junior H, Mendes Klumb E, et al. Neuroophthalmologic manifestations in systemic lupus erythematosus. Lupus. 2017 Apr;26(5):522-8. https:// doi.org/10.1177/0961203316683265

26. Zahid S, Iqbal M. Systemic Lupus Erythematosus Presenting as Optic Neuropathy: A Case Report. Cureus [Internet]. [cited 2021 Jan 24];11(6). https:// doi.org/10.7759/cureus.4806

27. García-Reynoso MJ, Veramendi-Espinoza LE, RuizGarcia HJ. Ascending Paresis as Presentation of an Unusual Association Between Necrotizing Autoimmune Myopathy and Systemic Lupus Erythematosus. Reumatol Clínica Engl Ed. 2014 May 1;10(3):183-6. https://doi.org/10.1016/j.reumae.2013.12.015

28. Tsukamoto $M$, Shimamoto $M$, Terashima $T$, Seta N. Aseptic Meningitis With Systemic Lupus Erythematosus: Case Report and Review of the Literature. Arch Rheumatol. 2018 Sep 4;34(1):108-11. https://doi. org/10.5606/ArchRheumatol.2019.7026

29. Magro Checa C, Cohen D, Bollen ELEM, van Buchem MA, Huizinga TWJ, Steup-Beekman GM. Demyelinating disease in SLE: Is it multiple sclerosis or lupus? Best Pract Res Clin Rheumatol. 2013 Jun 1;27(3):40524. https://doi.org/10.1016/j.berh.2013.07.010

30. Greenwood DLV, Gitlits VM, Alderuccio F, Sentry JW, Toh B-H. Autoantibodies in Neuropsychiatric Lupus. Autoimmunity. 2002 Jan;35(2):79-86. https:// doi.org/10.1080/08916930290016547

31. West SG, Emlen W, Wener MH, Kotzin BL. Neuropsychiatric Lupus Erythematosus: A lo-Year Prospective Study on the Value of Diagnostic Tests. Am J Med. 1995 Aug;99:153-63. https://doi.org/10.1016/S00029343(99)80135-1

32. Lampropoulos CE, Koutroumanidis M, Reynolds PPM, Manidakis I, Hughes GRV, D'Cruz DP. Electroencephalography in the assessment of neuropsychiatric manifestations in antiphospholipid syndrome and systemic lupus erythematosus. Arthritis Rheum. 2005 Mar;52(3):841-6. https://doi.org/10.1002/art.20957

33. Sibbitt WL, Sibbitt RR, Brooks WM. Neuroimaging in neuropsychiatric systemic lupus erythematosus. Arthritis Rheum. 1999 Oct;42(10):2026-38. https://doi. org/10.1002/1529-0131(199910)42:10\%3C2026::AIDANR2\%3E3.0.CO;2-J

34. Fernandes Moça Trevisani V, Castro AA, Ferreira Neves Neto J, Atallah ÁN. Cyclophosphamide versus methylprednisolone for treating neuropsychiatric involvement in systemic lupus erythematosus. Cochrane Musculoskeletal Group, editor. Cochrane Database Syst Rev [Internet]. 2013 Feb 28 [cited 2021 Feb 26]; Available from: http://doi.wiley.com/10.1002/14651858. CD002265.pub3. 